\title{
Distúrbios de Atitudes Alimentares e \\ Distorção da Imagem Corporal no Contexto \\ Competitivo da Ginástica Rítmica
}

\section{Eating Disorders and Body Image Distortion in the Rhythmic Gymnastics Competitive Context}

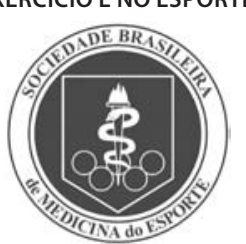

Artigo Original

\author{
José Luiz Lopes Vieira' \\ Helenice Zotto Amorim² \\ Lenamar Fiorese Vieira' \\ Adolpho Cardoso Amorim ${ }^{3}$ \\ Priscila Garcia Marques da Rocha ${ }^{4}$ \\ 1. UEM - Paraná. \\ 2. Unimep - São Paulo. \\ 3. Cesumar - Paraná. \\ 4. Faculdade Ingá/Uningá - Paraná.
}

Endereço para correspondência: Priscila Garcia Marques da Rocha Rua Luiz Fabeni, 35, Jd. Custódio 86990-000 - Marialva, PR - Brasil.

Tel.: + 55 (44) 3232-5792.

E-mail: pgmrocha@yahoo.com.br

Submetido em: 19/09/2007

Versão final recebida em: 14/04/2009 Aceito em: 27/07/2009

\begin{abstract}
RESUMO
Objetivo: Investigar a presença e a relação entre comportamentos sugestivos de transtornos de conduta alimentar e distorção da imagem corporal de atletas de ginástica rítmica participantes do contexto esportivo competitivo. Métodos: Participaram do estudo 48 atletas de ginástica rítmica, das categorias: infantil, juvenil e adulta e 48 estudantes do ensino fundamental e médio (controle). Para a identificação da presença de transtornos de conduta alimentar utilizou-se o Teste de Atitudes Alimentares (EAT-26) e, para determinar a insatisfação com a imagem corporal, o Body Shape Questionnaire (BSQ). Para a análise estatística utilizou-se o teste de Kruskal-Wallis ( $<0,05)$ e a correlação de Spearman. Resultados: Não houve diferenças estatísticas significativas entre o grupo de ginastas e o de estudantes na presença de transtornos de conduta alimentar; constatou-se correlação significativa entre o transtorno de conduta alimentar e distorção de imagem corporal para as ginastas da categoria infantil (10-12 anos) e juvenil (13-14 anos). A análise descritiva revela que 27,2\% (quatro ginastas/10-12 anos); 20,0\% (quatro ginastas/13-14 anos) apresentaram atitudes sugestivas de comportamento de transtorno de conduta alimentar. Duas ginastas de 10-12 anos (10,0\%), cinco ginastas de 13-14 anos (15\%) e as cinco ginastas acima 15 anos (100\%) apresentaram distorção da imagem corporal. Conclusão: As evidências apontam que a percepção comportamental e cognitiva das adolescentes em relação às exigências estéticas dos ambientes nos quais estão inseridas parece ser um fator primordial a ser considerado quando da presença de comportamentos sugestivos de transtorno de conduta alimentar e da distorção da imagem corporal.
\end{abstract}

Palavras-chave: ginastas, transtorno alimentar, imagem corporal.

\section{ABSTRACT}

Objective: The aim of this study was to investigate the presence and relationship between eating behavior disorders and body image distortion in rhythmic gymnastics' athletes who participate in a competitive sports context. Methods: 48 rhythmic gymnastics athletes from the junior and senior categories and 48 elementary and high school students (control group) participated in the study. For the identification of eating behavior disorder the Eating Attitude Test (EAT-26) was used and to determine body image dissatisfaction the Body Shape Questionnaire (BSQ). For statistic analysis the Kruskall-Wallis test $(p<0.05)$ and Spearman Correlation were used. Results: there were not statistical differences between the gymnastics group and the students group for eating behavior disorder; a significant correlation was noticed between eating behavior disorder and body image distortion for the athletes from younger junior (10-12 years old) and older junior (12-14 years old) sport categories. Descriptive analysis revealed that 27.2\% (4 gymnastics/10-12 years old); 20.0\% (4 gymnasts/13-14 years old) presented suggestive behavior of eating disorder. Two gymnasts aged 10-12 years old (10.0\%), five gymnasts aged 13-14 years old (15.0\%) and five 15 years old gymnasts (100\%) presented body image distortion. Conclusion: The evidence points that the adolescents' behavioral and cognitive perception related to the environment's esthetic demands in which they are inserted seem to be a main factor to be taken in consideration as for the presence of behavior that suggests eating disorder and body image distortion.

Keywords: gymnasts, eating disorders, body image. 


\section{INTRODUÇÃO}

A magreza tem sido adotada como o perfil ideal de beleza e de boa aceitação social, embora nem sempre o conceito de que magreza denote saúde (1). No contexto competitivo, o perfil corpóreo necessário vai muito além das ideologias implantadas pela sociedade ${ }^{(2,3)}$. Os atletas de alto rendimento estão submetidos a processos de treinamento e competição que geram a necessidade de manipular a alimentação e o peso corporal na tentativa de aperfeiçoar a performance ${ }^{(4)}$. Essa conjuntura está presente em todas as modalidades esportivas ${ }^{(5)}$, mas em especial se evidencia em modalidades que exigem baixo peso corporal e que envolvem atletas do sexo feminino, como a ginástica rítmica ${ }^{(6-9)}$.

Dentro desse contexto, surgem estudos que investigam o comportamento alimentar e como a percepção da imagem corporal de um indivíduo pode acarretar distúrbios como anorexia e bulimia(10-12), inclusive com foco em atletas ${ }^{(1,8-13)}$. No caso das atletas de ginástica rítmica, a imposição sobre o "corpo magro" e as restrições alimentares geram a sensação surreal de que nunca se está "magra" o suficiente ${ }^{(9)}$. Essa visão distorcida do próprio corpo pode acarretar uma série de distúrbios de comportamentos alimentares que não devem ser ignorados dentro do ambiente esportivo ${ }^{(6,7)}$.

Os transtornos de conduta alimentar como anorexia e bulimia nervosa parecem ser mais frequentes em indivíduos atletas do que em não atletas ${ }^{(14)}$. Essa alta prevalência foi observada em esportes que exigem baixo peso corporal como fator indispensável para o desempenho esportivo ${ }^{(15,16)}$.

Nas modalidades esportivas em que o baixo peso corporal e/ou baixo índice de gordura corporal são requisitos indispensáveis para a desempenho, em especial para atletas do gênero feminino, como é o caso da ginástica rítmica, a exigência de ser e estar magra desencadeia um processo lento, e muitas vezes não clínico, de ansiedade e estresse acerca do próprio corpo ${ }^{(17)}$. Acredita-se que ao agir de forma negativa sobre as dimensões psicológicas das atletas, o propósito de magreza distorce a imagem corporal ${ }^{(15)}$.

Embora pesquisadores ${ }^{(1,8,13-15)}$ tenham focado os distúrbios de atitudes alimentares no esporte, são escassas as evidências quanto ao momento da vida atlética em que surgem os transtornos de conduta alimentar e nem a relação com a distorção da imagem corporal, particularmente, em atletas de ginástica rítmica.

Dessa forma, é interessante destacar que a ginástica rítmica, assim como as demais modalidades esportivas, categoriza as atletas por faixas etárias. A identificação de transtorno de conduta alimentar, assim como a distorção da imagem corporal em diferentes momentos da carreira esportiva dessas ginastas, poderia contribuir para compreensão dos mecanismos que desencadeiam tais processos prejudiciais à saúde das atletas.

Sob essa perspectiva, este estudo se propôs a investigar a presença e a relação entre comportamentos sugestivos de transtornos de conduta alimentar e distorção da imagem corporal de atletas de ginástica rítmica (GR) participantes do contexto esportivo competitivo.

\section{MÉTODOS}

\section{Caracterização da amostra}

Este estudo caracteriza-se como transversal, tendo como premissa explorar a relação entre a presença de transtornos de atitudes alimentares e distorção de imagem corporal. A população deste estudo foi composta por todas as atletas de ginástica rítmica com idade entre 10 e 18 anos do Estado do Paraná. A amostra foi determinada de forma estratificada, devido às atletas pertencerem ao quadro de filiadas à Federação Paranaense de Ginástica Rítmica que preencheram os se- guintes requisitos para participar do estudo: ter praticado de forma intensiva a ginástica rítmica durante, no mínimo, dois anos consecutivos, e ter participado de pelo menos duas competições esportivas em nível estadual, nacional ou internacional. Dessa forma, a amostra do estudo foi constituída por 48 atletas, sendo 22 ginastas da categoria infantil, 21 da categoria juvenil e cinco da categoria adulta.

Para efeito de comparação, foi constituído por sorteio o grupo de estudantes do ensino fundamental e médio não atletas (controle) selecionado a partir das informações de dados pessoais obtidos das atletas de GR, usando como critérios: a idade, estar matriculadas em instituições de ensino público ou privado na mesma proporção das frequentadas pelas atletas de GR e não estar envolvidas em programa de esporte competitivo. A tabela 1 demonstra as características antropométricas das atletas de GR e das estudantes não atletas.

Tabela 1. Peso e altura das atletas de ginástica rítmica das categorias (infantil: 10-12 anos; juvenil: 13-14 anos; adulto: acima de 15 anos) e de estudantes não atletas.

\begin{tabular}{l|c|c|c|c|c|c}
\hline \multirow{2}{*}{ Amostra } & \multicolumn{3}{|c|}{$\begin{array}{c}\text { Atletas de ginástica rítmica } \\
(\mathbf{n}=\mathbf{4 8})\end{array}$} & \multicolumn{3}{c}{$\begin{array}{c}\text { Estudantes não atletas } \\
(\mathbf{n}=\mathbf{4 8})\end{array}$} \\
\hline Idade (anos) & $10-12$ & $13-14$ & Acima de 15 & $10-12$ & $13-14$ & Acima de 15 \\
\hline $\mathrm{N}$ & 22 & 21 & 5 & 22 & 21 & 5 \\
\hline Altura (cm) & $143 \pm 0,09$ & $155 \pm 0,06$ & $162 \pm 0,02$ & $149 \pm 0,11$ & $160 \pm 0,06$ & $164 \pm 0,07$ \\
\hline Peso (kg) & $31,9 \pm 5,01$ & $40,6 \pm 4,83$ & $50,6 \pm 3,43$ & $42,7 \pm 13,14$ & $49,0 \pm 7,22$ & $61,0 \pm 6,08$ \\
\hline
\end{tabular}

\section{Instrumentos de medida}

Para a identificação de transtornos de conduta alimentar foi utilizado o Teste de Atitudes Alimentares (EAT-26), contendo 26 questões com opções de resposta do tipo Likert (sempre, muito frequente, frequentemente, algumas vezes, raramente e nunca). A tradução do EAT-26 foi realizada por Nunes et al. (1998)(18,19). A avaliação das respostas é feita atribuindo-se três pontos para cada item em que foi marcada a resposta "sempre", dois pontos para "muito frequentemente" e um ponto para "frequentemente"; as demais respostas não são pontuadas. Quando o total de pontos obtidos é maior que 21, a presença de transtornos de conduta alimentar é positiva.

Para avaliar a satisfação com a imagem corporal foi utilizado o questionário Body Shape Questionnaire (BSQ), que foi traduzido por Cordás e Neves (1999)20 e validado para uma população de universitários brasileiros por Di Pietro (2002) ${ }^{21}$. O BSQ avalia a preocupação com a imagem corporal. O instrumento possui 34 questões do tipo Likert com seis opções de respostas: 1 - nunca; 2 - raramente; 3 - às vezes; 4 - frequentemente; 5 - muito frequente; e 6 - sempre. A classificação dos resultados é feita pelo total de pontos obtidos e reflete quatro grupos possíveis: (a) < 81 pontos: não preocupados com a imagem corporal (sem presença de distorção de imagem corporal); (b) 81 a 110 pontos: sem preocupação com a imagem corporal (leve distorção de imagem corporal); (c) 111 a 140 pontos: moderadamente preocupados (distorção de imagem corporal moderada); (d) > 140 pontos: extremamente preocupados com a imagem corporal (distorção de imagem corporal grave).

\section{Procedimentos e coleta de dados}

Inicialmente foram enviadas cartas-convites aos clubes para participarem da pesquisa; a partir do consentimento dos pais das atletas participantes de GR no respectivo clube, os dados foram coletados pelos pesquisadores. Os pesquisadores se deslocaram às cidades onde se localizam as equipes que consentiram em participar do estudo e 
os dados foram coletados no local de treinamento das atletas em dias e horários previamente combinados. Para a coleta dos dados das estudantes (controle), o procedimento adotado foi similar ao realizado com as atletas de GR, com solicitação de participação ao colégio e assinatura do termo de consentimento livre e esclarecido pelos pais. A carta-convite, o termo de consentimento livre e esclarecido para participação do estudo e o projeto da pesquisa foram submetidos à análise do Comitê Permanente de Ética em Pesquisa com seres humanos.

\section{ANÁLISE ESTATÍSTICA}

Os resultados estão apresentados através da estatística descritiva. Após verificação da não normalidade dos dados, optou-se pela estatística não paramétrica, na qual se utilizou o teste de Kruskal-Wallis para análise de variância em função das categorias das amostras; para a correlação entre as variáveis investigadas nos grupos amostrais foi utilizado o teste de Spearman. Os níveis de significância estatística foram estabelecidos em $p \leq 0,05$.

\section{RESULTADOS}

As principais características do perfil antropométrico identificadas foram peso e altura. Para verificar a conduta alimentar, foram utilizados os resultados do EAT (transtorno de conduta alimentar) e BSQ (distorção de imagem corporal). A tabela 2 apresenta os resultados dessas variáveis.

Tabela 2. Comparação das médias de peso, altura, transtorno na conduta alimentar (EAT) e distorção de imagem corporal (BSQ) de atletas de ginástica rítmica - PR e estudantes não atletas (controle).

\begin{tabular}{|c|c|c|c|c|}
\hline \multirow{2}{*}{ Idade } & \multirow{2}{*}{ Variável } & Atleta & Não atleta & \multirow{2}{*}{ p-valor } \\
\hline & & M dp & M dp & \\
\hline \multirow{4}{*}{ 10-12 anos } & Peso (kg) & $31,9 \pm 5,02$ & $42,7 \pm 13,1$ & $0,042^{*}$ \\
\hline & Altura $(\mathrm{cm})$ & $143,0 \pm 0,06$ & $149,0 \pm 0,08$ & 0,684 \\
\hline & EAT-26' & $11,9 \pm 12,1$ & $12,1 \pm 11,5$ & 0,345 \\
\hline & $\mathrm{BSQ}^{2}$ & $61,1 \pm 28,0$ & $75,4 \pm 34,0$ & 0,080 \\
\hline \multirow{4}{*}{ 13-14 anos } & Peso (kg) & $40,6 \pm 4,81$ & $49,0 \pm 7,22$ & $0,001^{* *}$ \\
\hline & Altura $(\mathrm{cm})$ & $155,0 \pm 0,09$ & $160,0 \pm 0,13$ & 0,145 \\
\hline & EAT- $26^{1}$ & $11,7 \pm 9,87$ & $14,7 \pm 10,5$ & 0,245 \\
\hline & $\mathrm{BSQ}^{2}$ & $89,6 \pm 39,0$ & $81,6 \pm 18,1$ & 0,689 \\
\hline \multirow{4}{*}{ Acima de 15 anos } & Peso (kg) & $50,6 \pm 3,42$ & $61,0 \pm 6,03$ & $0,002^{* *}$ \\
\hline & Altura $(\mathrm{cm})$ & $162,0 \pm 0,05$ & $164,0 \pm 0,65$ & 0,062 \\
\hline & EAT-26 ${ }^{1}$ & $13,4 \pm 3,53$ & $17,0 \pm 6,76$ & 0,986 \\
\hline & $\mathrm{BSQ}^{2}$ & $95,2 \pm 13,7$ & $125,2 \pm 15,5$ & 0,069 \\
\hline
\end{tabular}

Nota - ${ }^{1}$ EAT-26: Teste de Atitudes Alimentares

2 BSQ: Body Shape Questionnaire

${ }^{*} p<0,05$ e ${ }^{* *} p<0,01$

Observa-se na tabela 2 que existe diferença estatisticamente significativa na comparação entre as médias do peso corporal entre o grupo de atletas de GR e o grupo de controle. Na análise verificou-se que o grupo de controle apresentou peso corporal maior em todas as idades. Esses resultados expressos em valores absolutos são de 10,8kg na idade 10-12 anos; 8,4kg na idade 13-14 e 10,4kg na idade acima de 15 anos.

Após a comparação das médias de peso e altura corporal intergrupos (GR e de controle) nas diferentes faixas etárias, a tabela 3 apresenta a análise de variância para o peso corporal, altura e índice de massa corporal, tendo como referência o grupo amostral específico (variância intragrupos).
Tabela 3. Análise de variância entre as variáveis do grupo de atletas de ginástica rítmica e estudantes não atletas do ensino fundamental e médio.

\begin{tabular}{|c|c|c|c|c|c|}
\hline \multicolumn{2}{|c|}{ Variável } & \multirow{2}{*}{$\begin{array}{l}\text { Variância } \\
{ }^{* *} 0,00\end{array}$} & \multirow{2}{*}{\begin{tabular}{|c}
$10-12$ anos/ \\
$13-14$ anos \\
$* * 0,00$
\end{tabular}} & \multirow{2}{*}{$\begin{array}{c}\begin{array}{r}13-14 \text { anos/ } \\
+ \text { de } 15 \text { anos }\end{array} \\
\quad *^{* *} 0,00\end{array}$} & \multirow{2}{*}{$\begin{array}{c}\begin{array}{c}10-12 \text { anos/ } \\
+ \text { de } 15 \text { anos }\end{array} \\
* * 0,00\end{array}$} \\
\hline \multirow{4}{*}{$\begin{array}{l}\text { Atletas } \\
\text { ginástica } \\
\text { rítmica }\end{array}$} & Peso (kg) & & & & \\
\hline & Altura $(\mathrm{cm})$ & $* * 0,00$ & $* * 0,00$ & 0,20 & $* * 0,00$ \\
\hline & EAT-26 ${ }^{1}$ & 0,79 & 0,49 & 0,24 & 0,70 \\
\hline & $\mathrm{BSQ}^{2}$ & 0,25 & 0,89 & 0,37 & 0,61 \\
\hline \multirow{4}{*}{$\begin{array}{l}\text { Estudantes } \\
\text { não atletas }\end{array}$} & Peso (kg) & ${ }^{*} 0,01$ & 0,15 & 0,08 & ${ }^{* *} 0,00$ \\
\hline & Altura (cm) & ${ }^{*} 0,02$ & $* * 0,00$ & 0,70 & $* 0,01$ \\
\hline & Atitude alimentar & 0,69 & 0,09 & 0,27 & 0,68 \\
\hline & Imagem corporal & 0,25 & ${ }^{* *} 0,02$ & 0,13 & 0,96 \\
\hline
\end{tabular}

Nota - ' EAT-26: Teste de Atitudes Alimentares

${ }^{2}$ BSQ: Body Shape Questionnaire

A tabela 4 apresenta os resultados referentes ao percentual da presença de transtorno de conduta alimentar e distorção da imagem corporal de atletas de GR e as estudantes não atletas.

Tabela 4. Percentual da presença de transtorno na conduta alimentar e distorção da imagem corporal de atletas de GR e estudantes não atletas.

\begin{tabular}{|c|c|c|c|c|c|c|}
\hline \multirow[t]{2}{*}{$\begin{array}{l}\text { Idade } \\
\text { (anos) }\end{array}$} & \multicolumn{2}{|c|}{$\begin{array}{c}\text { Transtorno de } \\
\text { conduta alimentar } \\
\text { (EAT-26) }\end{array}$} & \multicolumn{4}{|c|}{ Distorção da imagem corporal (BSQ) } \\
\hline & Ausência & Presença & Ausência & Leve & Moderada & Grave \\
\hline \multicolumn{7}{|l|}{$10-12$} \\
\hline Atletas & $81,8 \%$ & $18,2 \%$ & $91,0 \%$ & - & $4,5 \%$ & $4,5 \%$ \\
\hline Não atletas & $76,2 \%$ & $23,8 \%$ & $59,5 \%$ & $18,0 \%$ & $18,0 \%$ & $4,5 \%$ \\
\hline \multicolumn{7}{|l|}{$13-14$} \\
\hline Atletas & $81,9 \%$ & $19,1 \%$ & $57,1 \%$ & $14,3 \%$ & $9,5 \%$ & $19,1 \%$ \\
\hline Não atletas & $81,9 \%$ & $19,1 \%$ & $47,7 \%$ & $23,8 \%$ & $23,8 \%$ & $4,7 \%$ \\
\hline \multicolumn{7}{|l|}{ Acima de 15} \\
\hline Atletas & $100,0 \%$ & - & - & $100,0 \%$ & - & - \\
\hline Não atletas & $100,0 \%$ & - & - & $20,0 \%$ & $60,0 \%$ & $20,0 \%$ \\
\hline
\end{tabular}

Nota-se que nos resultados englobando todas as adolescentes (96) tanto para as atletas de GR quanto às estudantes não atletas, 85,3\% não apresentaram transtorno de conduta alimentar e em 66,2\% das adolescentes foi identificada ausência de distorção de autoimagem. No entanto, o grupo de atletas e estudantes não atletas acima de 15 anos foi o que apresentou maior índice de distorção da imagem corporal (100\%), classificada em graus "leve", "moderado" e "grave".

Tabela 5. Correlação entre o transtorno de conduta alimentar (EAT) e distorção de imagem corporal (BSQ) em atletas de GR e estudantes não atletas (controle).

\begin{tabular}{c|c|c}
\hline & Atletas & Não atletas \\
\hline $10-12$ anos & $0,754^{*}$ & $-0,153$ \\
\hline $13-14$ anos & $0,442^{*}$ & 0,133 \\
\hline Acima de 15 anos & $-0,100$ & 0,359 \\
\hline Geral & $0,435^{* *}$ & $0,665^{* *}$ \\
\hline
\end{tabular}


Apesar do baixo "n" amostral do grupo de atletas e estudantes acima de 15 anos, os resultados demonstraram que não ocorreu correlação estatisticamente significativa entre presença de transtorno de conduta alimentar e distorção de imagem corporal. No entanto, para as categorias de ginastas de GR infantil (10-12 anos) e juvenil (13-14 anos) constataram-se correlações positivas estatisticamente significativas entre transtorno de conduta alimentar e distorção de imagem corporal, embora a correlação se tenha reduzido com o avanço da idade $\left(0,754^{* *}\right.$ nas ginastas "infantil" e 0,442* nas ginastas "juvenil").

Quando correlacionados o transtorno de conduta alimentar e a distorção de imagem corporal no total do quadro amostral, tanto para as atletas quanto para as estudantes não atletas confirmou-se a correlação positiva estatisticamente significativa $\left(0,435^{* *}\right.$ para as atletas e $0,665^{* *}$ para as estudantes não atletas).

\section{DISCUSSÃO}

A obtenção e manutenção do padrão corporal característico para cada modalidade esportiva implicam, em particular, o controle alimentar, para adequar seu corpo às exigências e demandas do ambiente esportivo competitivo. A sociedade geralmente conduz e interfere nos comportamentos coletivos, e emagrecer é considerado um elemento essencial da etiologia dos chamados distúrbios alimentares ${ }^{(1,3,22)}$, os quais, juntamente com fatores biológicos, psicológicos e familiares, acabam gerando preocupação excessiva com o corpo, medo anormal de engordar e níveis de ansiedade crescentes, acompanhados de alterações da percepção corporal ${ }^{(14,15)}$.

O peso corporal baixo das ginastas nas diferentes idades pesquisadas parece ser determinante para a participação na GR no contexto competitivo. Assim, essa modalidade esportiva suporta a noção de uma subcultura que amplia as pressões socioculturais pela magreza ${ }^{(1,5-9)}$. Os resultados deste estudo sugerem que o peso corporal é um requisito fundamental para que a ginasta pratique esse esporte, que é caracterizado pela leveza dos movimentos, pela arte do equilíbrio, flexibilidade e pelo domínio do corpo e, devido a isso, preconiza-se que as ginastas sejam mais magras do que outras meninas da mesma idade ${ }^{(16)}$.

O baixo peso corporal das atletas no contexto competitivo esportivo da ginástica rítmica parece ser requisito para as ginastas terem desempenho dos movimentos técnicos da modalidade, o que, do ponto de vista da eficácia mecânica, exige um determinado biótipo ${ }^{(6-12)}$. A GR é uma modalidade na qual a criança inicia a prática desde muito cedo (por volta dos cinco anos de idade) e já é solicitada a apresentar padrões corporais (peso e altura), qualificando um biótipo que possibilite que a GR continue sendo praticada após o período da infância ${ }^{(13)}$. Quando o corpo estético não é o adequado, devido o estereotipo magérrimo bem abaixo do peso ideal, se torna necessário um regime alimentar rigoroso com finalidades competitivas, conduta muito comum na ginástica rítmica ${ }^{(8)}$.

O perfil longilíneo e magro é um elemento essencial e imprescindível para atletas de GR de alto rendimento esportivo e, por consequência, para as faixas etárias que antecedem esse estágio da carreira esportiva; portanto, é prioridade que a magreza seja plenamente alcançada ${ }^{(11)}$. Essa tipologia corporal das atletas de GR se evidenciou nas ginastas paranaenses; em todas as faixas etárias (categorias esportivas), o peso corporal das atletas de GR foi estatisticamente menor do que o peso corporal do grupo de estudantes (controle). No entanto, a altura corporal do grupo de controle foi em todas as faixas etária superior ao do grupo de atletas de ginástica rítmica.

O perfil corporal que enfatiza o baixo peso necessário ao desempenho esportivo é relatado na literatura ${ }^{(4,7,10-23)}$, sugerindo que as atletas estão mais suscetíveis às opiniões da sociedade e pressões em relação ao desempenho, tornando-as altamente influenciáveis e com grandes possibilidades de desenvolver desordens alimentares ${ }^{(11)}$. Por outro lado, mesmo em ambiente de vida cotidiana e, principalmente, nas faixas etárias de 10-12 anos e 13-14 anos, os resultados do grupo de estudantes não atletas demonstraram pontuação maior do que as atletas de GR para a presença de transtornos de atitude alimentar. Sendo assim, a pressão social diária pelo corpo magro parece ter tanto impacto para surgimento de transtornos de conduta alimentar e distorção de imagem corporal nas adolescentes de forma geral, quanto o ambiente esportivo competitivo nas ginastas de GR.

Assim, a percepção da autoimagem corporal tem forte raiz na tendência de as sociedades ocidentais contemporâneas viverem atualmente sob o ideal de magreza e da boa forma física, cujo padrão se impõe especialmente para as mulheres, nas quais a aparência física representa uma importante medida de valor pessoal(24). O impacto desse padrão no comportamento revela-se no desejo generalizado por um corpo mais magro, e a discrepância entre o peso real e o ideal leva a um estado de constante insatisfação com o próprio corpo, causando distorções dessa imagem ${ }^{(10-13)}$.

Neste estudo, os resultados demonstraram que as atletas de GR e as estudantes não atletas mais jovens apresentaram maior dificuldade na adaptação à conduta alimentar, enquanto que a distorção de imagem se acentua com o avanço da idade. As atletas de GR apresentaram índices menores de distorção da imagem corporal do que as estudantes não atletas. Supõe-se que o padrão corporal exigido para o desempenho no ambiente da ginástica rítmica competitiva esteja próximo da estética delineada de "corpo magro" veiculado como o padrão corporal da menina/ adolescente.

Estudos ${ }^{(2,6-1)}$ relatam que vários são os fatores que desencadeiam os transtornos alimentares em indivíduos que têm uma carga de tarefa física acentuada, e também podem ter etiologia com a personalidade, dinâmica familiar e ambiente sociocultural. Esses fatores, se deixados para ser diagnosticados mais tarde, podem ser difíceis de ser detectados na sua origem e corrigidos em tempo de evitar maiores prejuízos ao indivíduo. Portanto, a percepção da imagem corporal distorcida manifestou-se tanto nas atletas de GR (16 atletas ou 33,3\%) como nas estudantes não atletas (25 estudantes ou 52,1\%).

Foi possível observar que nas atletas de GR e nas estudantes não atletas, a distorção da imagem corporal não estava associada à presença de transtorno na conduta alimentar; mas em todas (100\%) as adolescentes avaliadas com presença de transtorno de conduta alimentar também se constatou distorção de imagem corporal. Esses resultados remetem a um estudo em particular ${ }^{(17)}$ e mostram que apenas o ambiente esportivo não é o único indicador para o aparecimento de transtorno de conduta alimentar e da distorção de imagem corporal. As evidências apontam que a percepção comportamental e cognitiva individual das adolescentes em relação às demandas dos ambientes nos quais estão inseridas parece ser um fator primordial a ser considerado.

Estudos $^{(4,5,7-11)}$ corroboram a hipótese de que a distorção da imagem corporal seja incentivada por razões multifatoriais, psicológicas, ambientais ou individuais, e consequentemente, seja um importante fator de risco de patologias relacionadas com o desenvolvimento de transtorno de conduta alimentar, como a anorexia e a bulimia. Embora no ambiente esportivo as pressões ambientais e psicológicas pelo corpo magro sejam mais evidentes ${ }^{(6-10)}$, os resultados evidenciam que as adolescentes não atletas possuem a mesma chance de desenvolver processos de patologias alimentares do que as adolescentes atletas, ao se considerar que a percepção de autoimagem corporal é um fator de risco que não se deve ignorar (2,13-24). 


\section{CONCLUSÃO}

Para este estudo, as evidências encontradas indicam que o comportamento sugestivo para a presença de transtorno de conduta alimentar e a distorção da imagem corporal no ambiente esportivo competitivo parece ter probabilidade similar à de qualquer outro contexto social no qual a exigência pela estética corporal seja o principal fator de referência. Cada adolescente, seja atleta ou não, estará sujeita ao desenvolvimento desses tipos de transtornos ou distorção, sendo que a percepção comportamental e cognitiva individual das adolescentes sobre as demandas e exigências do ambiente parece ser o fator crucial nesse processo.

O comportamento sugestivo para o transtorno na conduta alimentar esteve presente em adolescentes ginastas e estudantes não atletas nas faixas etárias (categorias esportivas) menores (10-12 anos e 13-14 anos). Assim, a idade e o processo de adaptação às demandas ambientais atuam com maior intensidade sobre as pré-adolescentes e adolescentes com baixa faixa etária. Portanto, profissionais como psicólogos esportivos e nutricionistas devem constituir a equipe esportiva visando a prevenção ou detecção precoce da presença de transtornos de conduta alimentar.

Os resultados indicam a relação entre comportamento sugestivo para o transtorno de conduta alimentar e distorção de imagem corporal, principalmente em atletas de ginástica rítmica de baixa faixa etária (10-12 anos, categoria infantil e 13-14 anos, categoria juvenil). Mas todas as ginastas de GR que foram identificadas com comportamento sugestivo de transtorno de conduta alimentar também apresentaram distorção de imagem corporal (leve, moderada ou grave); por outro lado, retirando esse grupo, as demais ginastas que apresentam distorção de imagem corporal não apresentaram comportamento sugestivo de transtorno de conduta alimentar.

Para pesquisas futuras, sugere-se que sejam realizados estudos com foco em outras modalidades e que considerem fatores intervenientes como: 1) além do uso de questionários, o de entrevistas, pois a percepção individual sobre demandas negativas que levam ao desenvolvimento desses comportamentos não se evidencia apenas com a coleta de dados através de questionários; 2) o aumento do " $n$ " amostral quando da coleta de dados visando uma análise estatística mais apurada quando do contraste com atletas ou de outra categoria de comparação; 3) a avaliação de indivíduos (atletas ou não) de faixas etárias diferentes ou estudo longitudinal; esta sugestão tem como objetivo auxiliar na compreensão de como o processo de aparecimento de comportamento sugestivo de transtorno de conduta alimentar ou distorção da imagem corporal ocorre.

\section{AGRADECIMENTOS}

Agradecemos aos clubes e às escolas que consentiram em participar deste estudo, assim como aos técnicos, pais e professores que autorizaram as adolescentes a disponibilizar tempo para as coletas e ao trabalho da equipe de pesquisa.

Todos os autores declararam não haver qualquer potencial conflito de interesses referente a este artigo.

\section{REFERÊNCIAS BIBLIOGRÁFICAS}

1. Garner DM, Garfinkel PE. Socio-cultural factors in the development of anorexia nervosa. Psychol Med. 1980;10:647-56.

2. Reijonen JH, Pratt HD, Patel DR, Greydanus DE. Eating disorders in the adolescent population: an overview. J Adolesc Res. 2003;18:209-22.

3. Thome J, Espelage DL. Relations among exercise, coping, disordered eating and psychological health among college students. Eat Behav. 2004;5:337-51.

4. Patel DR, Grydanus DE, Pratt HD, Phillips EL. Eating disorders in adolescents athletes. J Adolesc Res. 2003:18:280-9.

5. Haase AM, Prapavessis H, Owens RG. Perfectionism, social physique anxiety and disordered eating: a comparison of male and female elite athletes. Psychol Sport Exerc. 2002;3:209-22.

6. Davis C. Body image, dieting behaviours, and personality factors: a study of high-performance female athletes Int J Sport Psychol. 1992;23:179-92.

7. Vardar E, Vardar SA, Kurt C. Anxiety of young female athletes with disordered eating behaviors. Eat Behav. 2007;8:143-7

8. Wilmore JH. Eating and weight disorders in the female athlete. Int I Sport Nutr. 1991;1:104-17.

9. Sundgot-Borgen J. Eating disorders in female athletes. Sports Med. 1994;17:176-88.

10. Smolak L, Murnen S, Ruble AE. Female athletes and eating problems. A metaanalysis. Int J Eat Disord. 2000;27:371-80.

11. Beals KA, Manore MM. Behavioral, psychological, and physical characteristics of female athletes with subclinical eating disorders. Int J Sport Nutr Exerc Metab. 2000;10:128-43.

12. Cupisti A, D'Alessandro C, Castrogiovanni S, Barale A, Morelli E. Nutrition survey in elite rhythmic gymnasts. J Sports Med Phys Fitness. 2000; 40:350-5.

13. Thompson AM, Chad KE. The relationship of social physique anxiety to risk for developing and eating disorder in young females. J Adolesc Health. 2002;31:183-9.
14. Garfinkel PE, Lin E, Goering P, Spegg C, Goldbloom DS, Kennedy S, et al. Bulimia nervosa in a Canadian community sample: prevalence and comparison of subgroups. Am J Psychiatry. 1995;152:1052-8.

15. Zmijewski CF, Howard MO. Exercise dependence and attitudes toward eating among young adults. Eat Behav. 2003:4:181-95

16. Georgopoulos NA, Markou KB, Theodoropoulou A, Vagenakis GA, Benardot D, Leglise M, Et al. Height velocity and skeletal maturation in elite female rhythmic gymnasts. J Clin Endocrinol Metab. 2001;86:5159-64.

17. Dale, KS, Landers, DM. Weight control in wrestling: eating disorders or disordered eating? Med Sci Sports Exerc. 1999;31:1382-9.

18. Nunes MAA, Pinheiro AP. Risco e prevenção em transtornos do comportamento alimentar. In Nunes MAA et al editors. Transtornos alimentares e obesidade. Porto Alegre: Artmed, 1998

19. Nunes MAA, Bagatini LF, Abuchaim ALG. O teste de atitudes alimentares (EAT-26) em adolescentes de Porto Alegre. Arq Psiq Psicot Psican. 1994;1:132-7.

20. Cordas TA, Neves JEP. Escalas de avaliação de transtornos alimentares. Rev Psiquiatr Clín. 1999;26:41-7.

21. Di Pietro MC. Validade interna, dimensionalidade e desempenho da escala BSQ - "Body Shape Questionnaire" em uma populaçäo de estudantes universitários [Dissertação de Mestrado]. São Paulo: Escola Paulista de Medicina da Universidade Federal de São Paulo, 2002.

22. Szabo A. Physical activity as a source of psychological dysfunction. In Biddle SH, Fox KR. Boutcher SH, editors. Physical activity and psychological well-being. London \& New York: Routledge, 2001.

23. Vieira JLL, Oliveira LP, Vieira LF, Vissoci JRN, Hoshino E, Fernandes, S. Distúrbios de atitudes alimentares e sua relaçăo com a distorção da auto-imagem corporal em atletas de judô do Estado do Paraná. Revista da Educação Física/UEM. 2008;17:177-84

24. Fleck SJ. Body composition of elite American athletes. Am J Sports Med. 1983;11:398-403. 